\title{
Performance of Web BaSed Microprocessor System FOR MEASUREMENT OF SOIL QUALITY PARAMETERS
}

\author{
Sechkin Remzi, Tsvetelina Georgieva, Nadezhda Paskova \& Plamen Daskalov
}
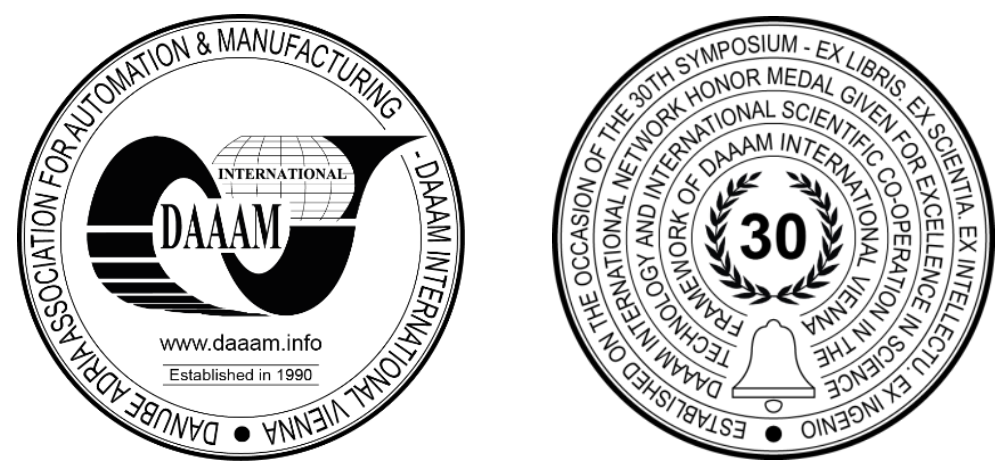

This Publication has to be referred as: Remzi, S[echkin]; Georgieva, T[svetelina]; Paskova, N[adezhda] \& Daskalov, P[lamen] I[vanov] (2019). Performance of Web Based Microprocessor System for Measurement of Soil Quality Parameters, Proceedings of the 30th DAAAM International Symposium, pp.1091-1096, B. Katalinic (Ed.), Published by DAAAM International, ISBN 978-3-902734-22-8, ISSN 1726-9679, Vienna, Austria

DOI: $10.2507 / 30$ th.daaam.proceedings. 152

\begin{abstract}
The paper presents web based microprocessor system for measurement of soil quality parameters ( $\mathrm{pH}$, electrical conductivity and soil moisture). The system is implemented by selecting suitable devices to measure the quantities and transmit and record the information based on Arduino platform. The software of the controller and a graphical user mobile applications interface have been developed for system setup, visualization and processing of the collected data. The developed program code is based on modules which makes it easy for using of additional sensors. Laboratory tests have been made to investigate the system's performance.
\end{abstract}

Keywords: Soil; Quality Parameters; Arduino platform.

\section{Introduction}

In agriculture, the production of vegetables is very important as it provides a significant part of the livelihood of the population and raw materials for the food industry.

Growing crops in a greenhouse has the advantage of all aspects of the production environment being controllable, as opposed to growing outdoors. The greenhouse protects the plants from adverse weather conditions and at the same time allows them to absorb the sunlight they need to feed them. One of the major factors affecting plant growth is the microclimate. Different types of crops have different optimum temperature and humidity of cultivation and they can be different for the soil and above ground part of the environment, as well as for different vegetation stages. For plant development and high yields, it is important to monitor soil moisture, electrical conductivity and soil $\mathrm{pH}$.

Soil moisture is the main indicator for assessing agricultural drought. Soil moisture levels can be expressed in terms of soil water content or water potential. The drier soil - the greater its water potential and the more difficult it is to extract water from the soil, on the other hand, excess moisture impairs the aeration of the soil, which impedes root respiration and the development of soil microorganisms. Measurement of water content in soil is performed by two methods: direct and indirect. In direct soil samples are taken and measurements and calculations are carried out in a laboratory using a precision balance and furnace, and in indirect soil moisture is obtained indirectly, for example as a result of the relationship between soil moisture and its dielectric constant, using capacitive sensors. 
The soil reaction is denoted by the $\mathrm{pH}$ index. This index is a number that reflects the concentration of hydrogen ions in the soil solution. Soil pH has an important role in the proper growth of crops and yields. The main reason for adjusting this parameter is that nutrient absorption depends on it. The optimum $\mathrm{pH}$ is different for different crops. The effect of soil $\mathrm{pH}$ on crop cultivation is significant. The soil $\mathrm{pH}$ factor influences many physical, chemical and biological reactions required for the survival, growth and yield of crops. The absorption of nutrients is strongly influenced by $\mathrm{pH}$ and is one of the most important reasons for regulating soil $\mathrm{pH}$. With increasing soil acidity, calcium carbonates dissolve and impair the physical properties of the soil. High acidity adversely affects plants, decreases productivity and often causes plants to die.

Soil electrical conductivity is used to characterize various soil properties such as residual moisture, salinity, nutrient concentration, and more. Soil conductivity correlates strongly with particle size and soil structure. Soil salinity is extremely important during sprouting and germination. The electrical conductivity of the soil solution or extract shows the concentration of the total soluble salts in the solution, thus reflecting the degree of salinity of the soil.

For the proper cultivation of plants in greenhouses, it is important to monitor and control the basic quality parameters. In recent years, various sensors have been widely used to monitor quality parameters in agriculture.

There are different types of methods and microprocessor systems for measuring non-electrical quantities for soil quality analysis. In [1] presents the possibility for using spectral data analysis for measuring of soil nitrogen. The prediction models between soil nitrogen and NIR spectral reflectance are presented using three chemometrics methods. Six wavebands from 1100 to $2400 \mathrm{~nm}$ are used for predicting the soil nitrogen content with wavelet analysis [2]. In [3] is presented neural network model of spectral characteristics for prediction of soil chemical and physical properties. Remote sensing based also on spectral wavebands is used for correlation of $\mathrm{pH}$ and electrical conductivity with reflectance band in [4]. Imaging spectroradiometer with moderate resolution is used for producing of soil salinity map in [5]. Vis and NIR spectral analysis are also used for total carbon and nitrogen prediction in the soils [6]. Digital image analysis is also used as a tool for soil analysis. In [7] is presented a method for classification of soil variations with analysis of color images. The results are slightly lower than traditional methods. RGB color space is used for determination of soil $\mathrm{pH}$ values in [8]. The system which used these kind of methods are expensive and required time for data processing and analysis.

The paper presents a low - cost microprocessor system for web monitoring of soil main quality parameters. The system is implemented by selecting suitable devices to measure the quantities and transmit and record the information based on Arduino platform. The software of the controller and a graphical user mobile applications interface have been developed for system setup, visualization and processing of the collected data. The future research will be focus on accuracy assessment of this system with traditional reference devices in real conditions.

\section{Design and development of a system for monitoring of basic quality soil parameters}

\subsection{Microprocessor system design}

A microprocessor system has been designed to measure basic air and soil parameters, calculate the required amount of fertilizer need to the plants and visualize the obtained values. Sensor's information is transferred to the control computer center. Appropriate calculations are made and the fertilization and irrigation processes of the plants are managed. Watering the plants is based on the information from the moisture sensor. The fertilization process requires preliminary experiments to determine exactly how and by what type it should be added. The most important elements for the growth of fertilizer plants are nitrogen $(\mathrm{N})$, phosphorus $(\mathrm{P})$ and potassium $(\mathrm{K})$, which is why they are called basic nutrients or macronutrients. These elements are usually added to the soil by fertilizing.

The basic block diagram of the system is shown on figure 1 .

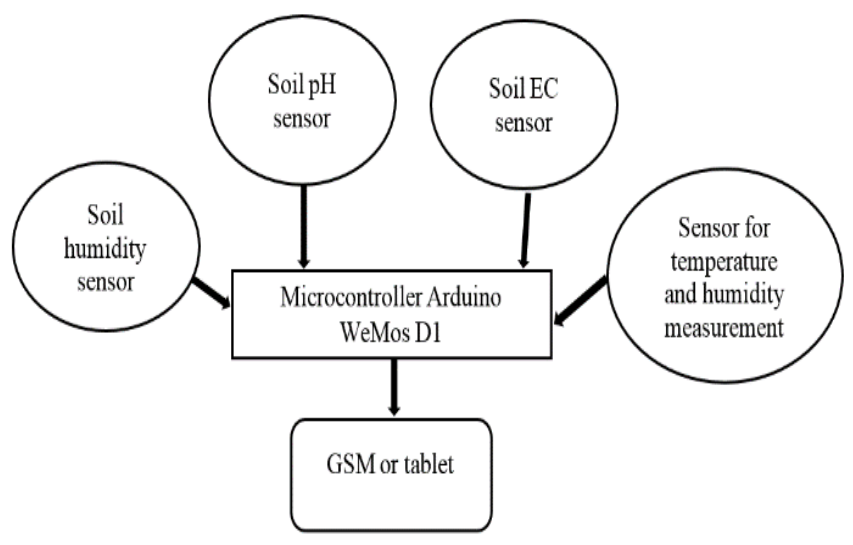

Fig. 1. Block diagram of the microprocessor system for air and soil parameters measurement 
The following components are used to design the system:

$>$ Microcontroller Arduino WeMos

Arduino WeMos (figure 2) is open source microcontroller development board. WeMos-D1 is based on the ESP8266$12 \mathrm{Wi}-\mathrm{Fi}$ microprocessor based on the Arduino UNO. The advantage of this micro controller is that it has a built-in wi-fi module. The board is managed using a set of instructions in the Arduino (Wiring based) programming language and the Arduino Software (Processing based) development environment.

Instead of using one of the Atmel / AVR series microcontrollers, the board uses the ESP8266EX chip. Some of the features of the ESP8266EX chip include:

- 32-bit RISC processor

- $80 \mathrm{MHz}$ clock speed

- 64kb RAM

- 96kb Data RAM

- 4-8MB flash memory

- Built-in WiFi

- Operating voltage - 3.3V

- Digital I / O Pins - 11.

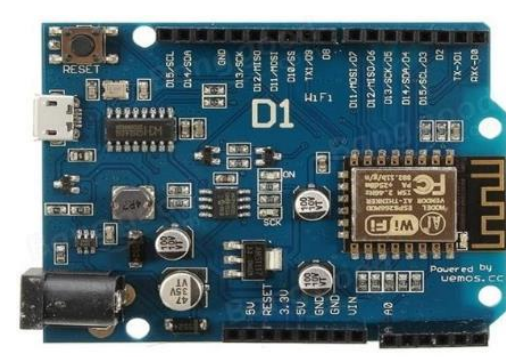

Fig. 2. Arduino WeMos

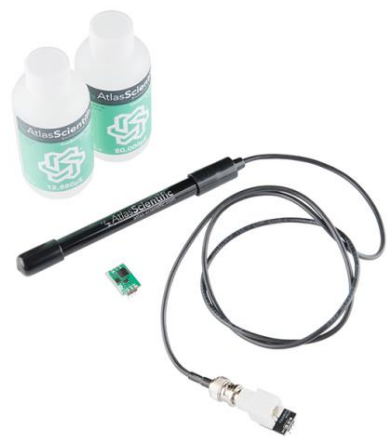

Fig. 4. Sensor for electrical conductivity

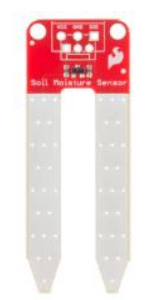

Fig.5. Soil moisture sensor

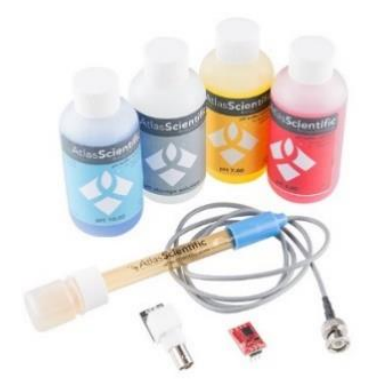

Fig. 3. PH sensor and its calibration solutions

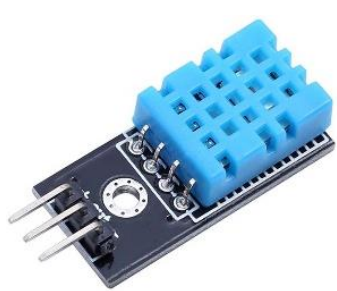

Fig. 6. Sensor for temperature

$>$ Sensor for $\mathrm{pH}$ measurement

The technical characteristics of the $\mathrm{pH}$ sensor (figure 3 ) are:

-pH Range: 0-14 (Na+ error at $>12.3 \mathrm{pH})$;

-Speed of Response: $95 \%$ in 1 second;

-Isopotential point: $\mathrm{pH} 7.00(0 \mathrm{mV})$;

-Offset: +/- $0.20 \mathrm{pH}$;

-3 buffer solutions and storage solution;

-38400 baud rate default.

Figure 2 shows the calibration solutions of the $\mathrm{pH}$ and the sensor. The solutions are:

-125ml Red Buffer Solution - pH 4.0;

-125ml Yellow Buffer Solution - pH 7.0;

$-125 \mathrm{ml}$ Blue Buffer Solution - pH 10.0;

$-125 \mathrm{ml}$ Storage Solution.

$>$ Sensor for measuring electrical conductivity

The technical characteristics of the electrical -conductivity meter presented in figure 4 are:

- Measuring Surface: Platinum black coated platinum;

- Max Temperature: 0-70 oC; 
- Conductivity readings $+/-2 \mu \mathrm{s} / \mathrm{cm}$;

- Full Conductivity range from $0.55 \mu \mathrm{s} / \mathrm{cm}$ to $500,000+\mu \mathrm{s} / \mathrm{cm}$.

Soil Moisture Sensor

The soil moisture sensor (figure 5) is a simple breakout for measuring the moisture in soil and similar materials. The soil moisture sensor is pretty straight forward to use. The two large exposed pads function as probes for the sensor, together acting as a variable resistor.

Sensor for measuring temperature and humidity

The DHT 11 (figure 6) is a temperature and humidity sensor with a calibrated digital signal output. This sensor includes the humidity measurement of a resistive component type and a temperature measurement component and is connected to an 8-bit microcontroller. DHT11 ranges and accuracy are the following:

- Humidity: 20-90\% RH;

- Humidity Accuracy: $\pm 5 \%$ RH;

- Temperature range: $0-50{ }^{\circ} \mathrm{C}$;

- Temperature accuracy: $\pm 2 \%{ }^{\circ} \mathrm{C}$;

- Operating voltage: $3 \mathrm{~V}$ to $5.5 \mathrm{~V}$.

\subsection{Developing a graphical user interface for a mobile web application.}

The graphical user interface (GUI) is developed for managing both the initialization of the module and the flow of information received from it. Through its software, the sensor network verifies the accuracy of the data, records it in a database and presents it to the user in an appropriate form. In this way, the user is provided with access to chronologically recorded data with corresponding correlations between them, for example, the change of concentration values over a period of time.

Through a properly developed graphical interface, the development platform provides the recorded data, through the adjacent periphery (monitor, projector) or remotely via a local or global network.

The main task of the GUI is to enable the user to view, produce reports, build statistical graphs and tables with the recorded data from the system.

Remote XY environment was selected to develop a graphical user interface.

RemoteXY is an easy way to create and use a mobile graphical user interface to control your smartphone or tablet. The connection between the controller and the mobile device is made through bluetooth; wifi client and hotspot; Ethernet over IP or URL or Internet through cloud server.

Supported Communication Modules are Bluetooth HC-05, HC-06 or compatible; WiFi ESP8266 or Ethernet board W5100. Supported mobile operating systems are Android and IOS.

Figure 7 shows the front panel of the developed graphical user interface for displaying information from the sensor for measuring temperature and humidity in a greenhouse, executed in RemoteXY software environment. The panel shows the measurements of air temperature and humidity. There are two buttons "Watering" and "Fertilization" which open another applications for displaying the values for watering and fertilization monitoring of the soil.

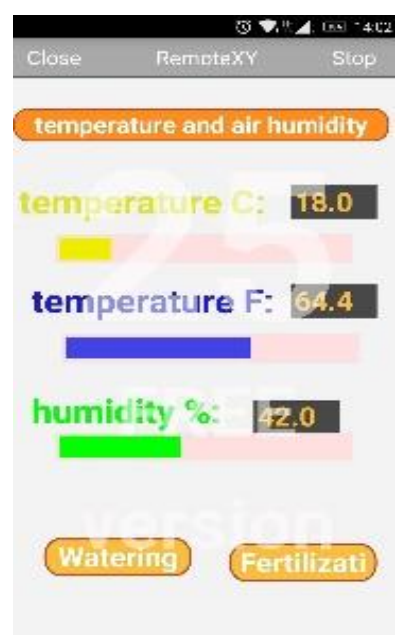

Fig. 7. Front panel of the developed graphical user interface

\section{Experimental test of the developed microprocessor system for monitoring the basic quality parameters}

The system was tested in a greenhouse layout with flower pots.

Figure 8 shows the system which is presented in working order, with sensors included and pump to the water tank. 


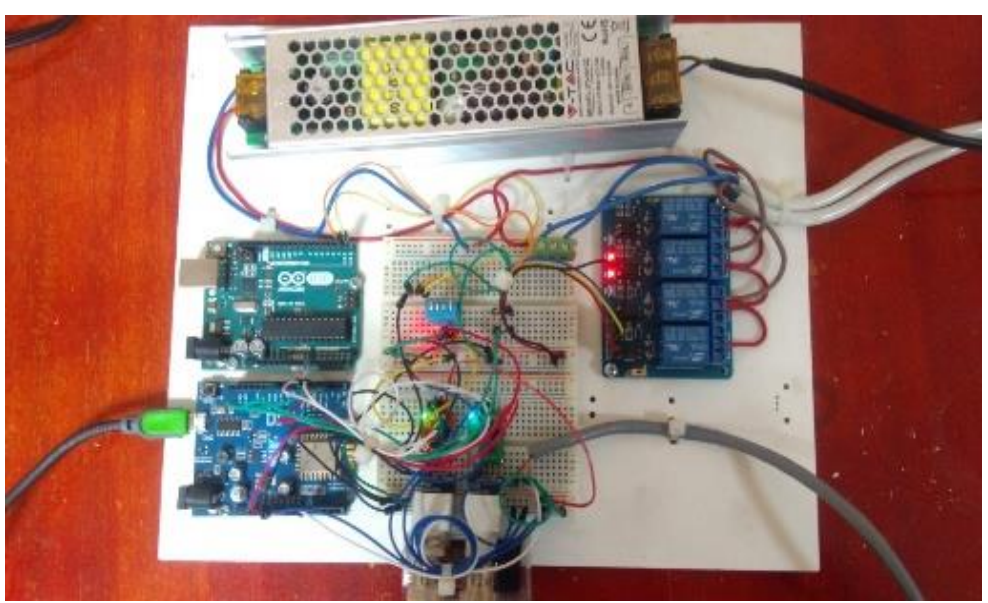

Fig. 8. Testing of the developed system

The result which is displayed in the command window of the program is presented on figure 9. It visualise the information from sensors for measuring electrical conductivity and soil $\mathrm{pH}$ in the ARDUINO IDE software platform

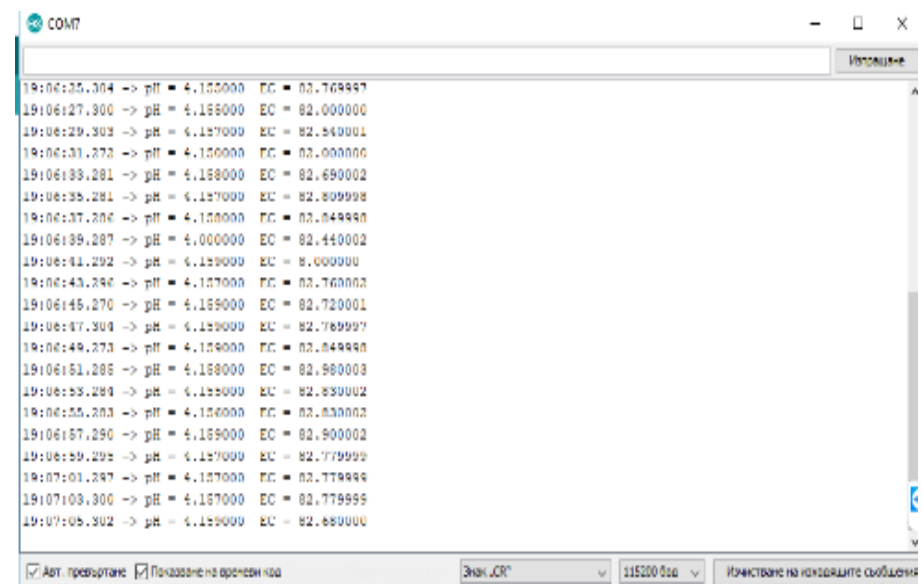

Fig. 9. Visualization of information from sensors for measuring electrical conductivity and soil $\mathrm{pH}$ in the ARDUINO IDE software platform

Data visualization takes place in a developed graphical user interface in RemoteXY. Figure 10 and figure 11 show the type of graphical user interface developed by the system for visualizing the information from the soil moisture content and sensor for electrical conductivity measurement in a greenhouse, executed in RemoteXY software environment.

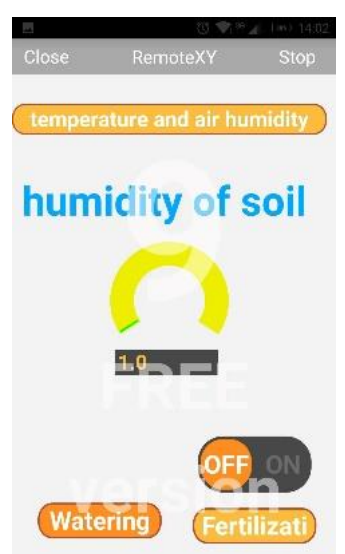

Figure 10. Display of information from the soil moisture sensor

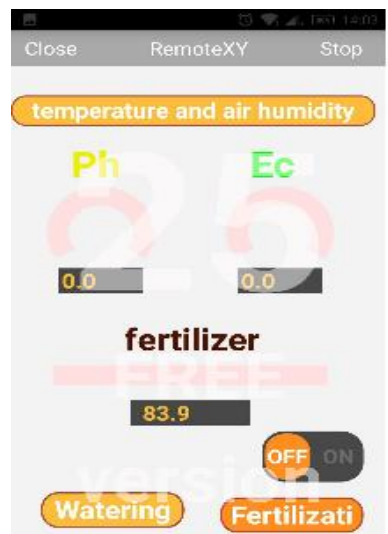

Figure 11. Visualization of information from sensors for $\mathrm{pH}$ measurement and electrical conductivity of soil

In the future developed system will be assess in the real time and conditions in the greenhouses. 


\section{Conclusion}

The existing systems which are used for measurement of soil parameters are expensive and required time for data processing and analysis. A low-cost system for measuring and wireless transmission of values of soil quality indicators ( $\mathrm{pH}$, electrical conductivity and soil moisture) and temperature and humidity of the greenhouse has been developed. The physical implementation of the system is implemented by selecting appropriate devices to measure the quantities, transmit and record the information. The software of the controller participating in the system and a graphical user interface have been developed for system setup, visualization and processing of the collected data. Laboratory tests have been made to assess the system's performance. The small size of the sensor modules makes it easy to place them in different locations. Their low-energy mode allows for longer battery life, if needed. The program code developed is modular, which makes it easy to add additional sensor modules, but also reduces system performance. Adding many endpoints requires additional optimizations over time. In the future developed system will be assess in the real time and conditions in the greenhouses.

\section{Acknowledgments}

The study was supported by contract of University of Ruse “Angel Kanchev”, № BG05M2OP001-2.009-0011-C01, " Support for the development of human resources for research and innovation at the University of Ruse "Angel Kanchev". The project is funded with support from the Operational Program " Science and Education for Smart Growth 2014 - 2020 " financed by the European Social Fund of the European Union.

The research is supported by a contract with Ruse University "Angel Kanchev" under NSF - 2019 EEA - 04, "Research of the influence of external factors on the accuracy of basic soil parameters measurement with a low-cost microprocessor system".

\section{References}

[1] Xiao, S.; He, Y.; Dong T. \& Nie, P. (2018). Spectral Analysis and Sensitive Waveband Determination Based on Nitrogen Detection of Different Soil Types Using Near Infrared Sensors, Sensors, Vol. 18, 523; doi:10.3390/s18020523

[2] Zhang, Y.; Li, M.Z.; Zheng, L.H.; Zhao, Y. \& Pei, X.S. (2016). Soil nitrogen content forecasting based on real-time nir spectroscopy. Comput. Electron. Agric. Vol. 124, 29-36.

[3] Aitkenhead, M.J.; Coull, M.C. \& Towers, W. (2012). Predicting soil chemical composition and other soil parameters from field observations using a neural network. Comput. Electron. Agric. Vol. 82, 108-116

[4] Bai, L.; Wang, c.; Zang, S.; Zhang, Y.; Hao, Q. \& Wu, Y. (2016). Remote Sensing of Soil Alkalinity and Salinity in the Wuyu'er-Shuangyang River Basin, Northeast China, Remote Sensing. Vol. 8, 163; doi:10.3390/rs8020163

[5] Shamsi, F.; Zare, S. \& Abtahi, S. (2013). Soil salinity characteristics using moderate resolution imaging spectroradiometer (MODIS) images and statistical analysis. Arch. Agron. Soil Sci. Vol. 59, 471-489.

[6] Kawamura, K.; Tsujimoto, Y.; Rabenarivo, M.; Asai, H.; Andriamananjara, A. \& Rakotoson, T. (2017). Vis-NIR Spectroscopy and PLS Regression with Waveband Selection for Estimating the Total C and N of Paddy Soils in Madagascar. Remote Sensing, Vol. 9, 1081, https://doi.org/10.3390/rs11091081

[7] Aydemira, S.; Keskinb, S. \& Drees, L. (2004). Quantification of soil features using digital image processing (DIP) technique. Geoderma, Vol. 119, 1-8, https://doi.org/10.1016/S0016-7061(03)00218-0

[8] Sudha, R.; Aarti, S.; Anitha, S. \& Nanthini, K. (2017). Determination Of Soil Ph And Nutrient Using Image Processing. International Journal of Computer Trends and Technology (IJCTT) - Special Issue April, 2017, 58-61, ISSN: $2231-2803$ 